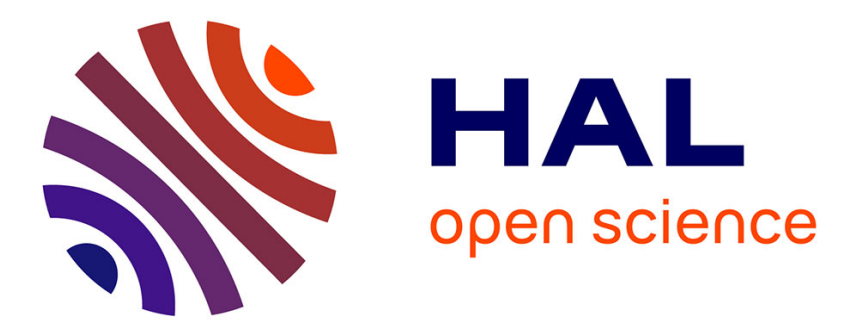

\title{
A micro-nano-rheometer for the mechanics of soft matter at interfaces
}

Léo Garcia, Chloé Barraud, Cyril Picard, Jérôme Giraud, Elisabeth Charlaix, Benjamin Cross

\section{- To cite this version:}

Léo Garcia, Chloé Barraud, Cyril Picard, Jérôme Giraud, Elisabeth Charlaix, et al.. A micro-nanorheometer for the mechanics of soft matter at interfaces. Review of Scientific Instruments, 2016, 87 (11), pp.113906. 10.1063/1.4967713 . hal-01581974

\section{HAL Id: hal-01581974 \\ https://hal.science/hal-01581974}

Submitted on 5 Sep 2017

HAL is a multi-disciplinary open access archive for the deposit and dissemination of scientific research documents, whether they are published or not. The documents may come from teaching and research institutions in France or abroad, or from public or private research centers.
L'archive ouverte pluridisciplinaire HAL, est destinée au dépôt et à la diffusion de documents scientifiques de niveau recherche, publiés ou non, émanant des établissements d'enseignement et de recherche français ou étrangers, des laboratoires publics ou privés. 


\title{
A micro-nano-rheometer for the mechanics of soft matter at interfaces
}

\author{
Léo Garcia, ${ }^{1}$ Chloé Barraud, ${ }^{1}$ Cyril Picard, ${ }^{1}$ Jérôme Giraud, ${ }^{1}$ Elisabeth Charlaix, ${ }^{1}$ and Benjamin Cross $^{1}$
}

Université Grenoble Alpes, CNRS, LIPhy, 38041 Grenoble Cedex 9, France

(Dated: 21 October 2016)

We present a nano-rheometer based on the dynamic drainage flow between a sphere and a plane from bulk regime to highly confined regime. The instrument gives absolute measurements of the viscosity of simple liquids in both regime. For complex fluids, the measurements involves the viscosity and the elastic modulus. The device operates on distances ranging over four orders of magnitude from 1 nanometer to 10 micrometers, bridging rheological properties from the macroscopic to the molecular scale. This allows to measure an hydrodynamic or visco-elastic boundary condition and to explore the causes of the boundary condition at the microscopic level.

\section{INTRODUCTION}

The equilibrium and dynamical properties of confined fluidic or soft systems are of central interest in many industrial and environmental applications, such as friction and lubrication, transport in porous media, nano-pores or membranes, adhesion and mechanics of coatings, soft nano-composites, bio-engineering materials, etc... Fundamental questions relate to the understanding of interfacial mechanics, transport, and their coupling to surface interactions. Surface Force Apparatii are invaluable tools for probing soft matter properties at nanoscale ${ }^{1}$. Beyond seminal contributions on interactions, organization, and phase transitions in thin liquid films, the extension of the technique to dynamics forces has shown its ability to unravel interfacial hydrodynamics, and more recently, the non-contact mechanics of soft objects ${ }^{2-11}$. The wellcontrolled surface geometry and properties in SFA enables the proof-of-concept of methods further extended to the near-field probe techniques such as $\mathrm{AFM}^{12}$.

In this article, we describe a micro-nano-rheometer based on Dynamic Surface Force measurements. The devices operates in a wide range of confinement from $10 \mu \mathrm{m}$ to the molecular size, enabling us to bridge the continuum scale to the molecular scale. While the confinement varies continuously, the quasi-static and the rheological properties of soft or fluid layers, are measured in the framework of the linear response for frequencies between $10 \mathrm{~Hz}$ and $400 \mathrm{~Hz}$, the amplitude of the gap oscillation ranging between $1 \mathrm{pm}$ to $40 \mathrm{~nm}$. Whereas common rheometers need to assume the boundary condition near the wall, our dynamic SFA-based rheometer provides independent informations on bulk behavior and boundary condition, and can also be used to investigate the mechanical properties of the confining geometry without contact.

\section{PRINCIPLE AND OVERVIEW OF CAPABILITIES}

The principle of the instrument is sketched in the inset of figure 1 . The fluid system is confined in a confined geometry, between a sphere and a plane (crossed cylinders or two spheres are also possible). The distance $d(t)$ between the apex of the sphere and the plane is actuated by a home-made device as:

$$
d(t)=D+h_{o} \cos (\omega t+\phi)
$$

The average distance $D$ is varied quasi-statically during an experiment, in a range from $10 \mu \mathrm{m}$ down to contact. The harmonic oscillation of amplitude $h_{o} \ll D$ drives a squeeze flow in the liquid film. It gives access to the rheological properties in the linear regime limit, at very low shear. The force exerted on the surfaces is written as follow: $F(t)=F(D)+f \cos (\omega t+\psi)$. The zero-frequency component $F(D)$ is the quasi-equilibrium force at the distance $D$. From the harmonic component of the force $f_{\text {dyn }}=f \cos (\omega t+\psi)=\mathcal{R} e\left[f e^{i(\omega t+\psi)}\right]$ and from the harmonic gap oscillation $h_{d y n}=\mathcal{R} e\left[h_{o} e^{i(\omega t+\phi)}\right]$, is defined the mechanical impedance:

$$
Z(D, \omega)=\frac{f e^{i \psi}}{h_{o} e^{i \phi}}=Z^{\prime}+j Z^{\prime \prime}
$$

$Z^{\prime}$ corresponds to mechanical response in phase with the displacement and gives access to the elastic response of the system whereas $Z^{\prime \prime}$ corresponds to the component in phase with the velocity $\dot{h}_{d y n}$ and gives access to the dissipation in the system.

The case of a newtonian liquid confined between rigid walls with a no-slip boundary condition constitue a reference situation. In this framework and for distances $D \ll R$, the mechanical impedance of the liquid film is ${ }^{13}$ :

$$
Z=Z_{e q}^{\prime}+j \frac{6 \pi \eta \omega R^{2}}{D}
$$

with $Z_{e q}^{\prime}=d F(D) / d D$ the derivative of the equilibrium force, $\eta$ the liquid bulk viscosity, $\mathrm{R}$ the sphere radius, and $\omega / 2 \pi$ the drive frequency. Thus $1 / Z^{\prime \prime}$ gives access independently to the liquid viscosity and to the hydrodynamic thickness, i.e. the position of the no-slip plane.

To go beyond this initial reference situation, the three conditions can be relaxed. First, let's consider the case of a visco-elastic liquid flowing with the same no-slip boundary condition between rigid walls. The eq.(2) can be generalized straightforwardly by using the complex viscosity $\eta^{*}=\eta-i G / \omega$, with $G$ the shear elastic modulus ${ }^{14}$. The visco-elastic shear modulus of the liquid at the driving 


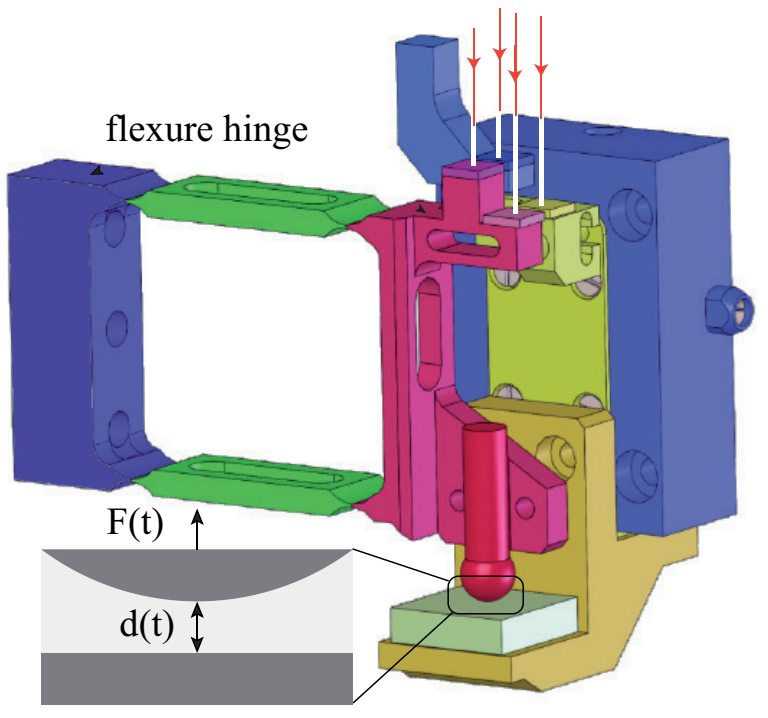

FIG. 1: Scheme of the nano-rheometer. The blue parts are fixed during an experiement. The red parts move

all-together when a force is applied to the sphere,

thanks to the flexure hinge. The yellow parts move

together when the piezoelectric device is actuated. Two mirror pairs receive the beams of the force

interferometer and of the sphere-plane displacement interferometer.

frequency is measured by the slope of the linear variation of $1 / Z^{\prime}$ with respect to $D$.

Second, let's consider that the boundary condition differs from a no-slip. The boundary condition has a signature on the mechanical impedance. In case of a Navier's partial slip $\lambda v_{\text {slip }}=\eta \partial v /\left.\partial z\right|_{\text {wall }}$ with $\lambda$ the fluid friction coefficient at the boundary ${ }^{15}$, the viscous damping writes $^{16,17}$ :

$$
\begin{array}{r}
Z=j \frac{6 \pi \eta \omega R^{2}}{D} f_{\text {slip }}\left(\frac{b}{D}\right) \quad b=\frac{\eta}{\lambda} \\
f_{\text {slip }}\left(\frac{b}{D}\right)=\frac{D}{3 b}\left[\left(1+\frac{D}{6 b}\right) \ln \left(1+\frac{6 b}{D}\right)-1\right]
\end{array}
$$

Moreover, this expression also holds in the case of a viscoelastic liquid ${ }^{18}$, using the complex slip length $b^{*}=\eta^{*} / \lambda$. At large distance, a seconde order Taylor expansion of eq.(4) gives the macroscopic behavior of the hydrodynamic impedance:

$$
D \gg\left|b^{*}\right| \quad \frac{6 \pi \omega R^{2}}{Z} \simeq j \frac{D+2 b^{*}}{\eta^{*}}=\frac{j D}{\eta^{*}}+\frac{2 j}{\lambda}
$$

Thus the real and imaginary components of $1 / Z$ at a large scale $D \gg\left|b^{*}\right|$ provide an independent evaluation of the visco-elastic modulii, the location of the flow boundary, and the boundary friction. At smaller scale, the full analytic expression (4) allows one to bridge the scales and quantify how the dynamics of the confined fluid deviates from the macroscopic rheological picture in terms of bulk and boundary properties.
Third, the elasticity of the confining geometry has an impact on the mechanical impedance. This effect provides a way to measure, without contact, the elastic modulus of the confining media or of thin coatings ${ }^{11,19}$. The elasto-hydrodynamic (EH) impedance taking into account the elastic deflection of the confining media writes $^{19}$ :

$$
Z=\frac{d F(D)}{d D}+\frac{6 \pi \eta \omega R^{2}}{D} g_{E H}^{*}\left(D / D_{c}\right)
$$

with $D_{c}$ an elasto-hydrodynamic distance depending on the coating thickness and modulii, the driving frequency, and $g_{E H}^{*}\left(D / D_{c}\right)$ a complex factor ${ }^{19}$. For homogeneous confining media of reduced Young modulus $E^{*}=E / 2\left(1-\nu^{2}\right)$ with $E$ the Young modulus and $\nu$ the Poisson ratio, the elasto-hydrodynamic distance is $D_{c}=8 R\left(\eta \omega / E^{*}\right)^{2 / 3}$. At large distance $D \gg D_{c}$, the asymptotic behavior of $g_{E H}^{*}\left(D / D_{c}\right)$ allows to reduce the inverse of the $\mathrm{EH}$ impedance to ${ }^{19}$ :

$$
\frac{1}{Z} \simeq-\frac{j D}{6 \pi \eta \omega R^{2}}+\frac{3 \pi}{32 E^{*} \sqrt{2 R D}}
$$

And in case of layered solids made of a thin soft coatings of thickness $\tau$ and shear modulus $G_{s}$ on a stiff support. The eq. (8) holds when $D \gg D_{c}=\tau\left(4 \eta \omega / 3 G_{s}\right)^{1 / 3}$

$$
\frac{1}{Z} \simeq-\frac{j D}{6 \pi \eta \omega R^{2}}+\frac{2 \tau^{3}}{9 \pi G_{s} R^{2} D^{2}}
$$

Thus, when the liquid used is fully Newtonian, the real part of $1 / Z$ provides a non-contact measurement of the confining media elasticity.

\section{DESIGN AND PERFORMANCES}

\section{A. Mechanical design}

A schematic view of the nano-rheometer is shown in figure 1 . The plane surface is actuated via a vertical translation stage composed of two parts. First a M126PI DC motor is used to reduce the gap between the plane and the sphere up to 10 microns by displacements from $1 \mu \mathrm{m}$ to $25 \mathrm{~mm}$. Second a piezoelectric actuator PI LISA753 of $15 \mu \mathrm{m}$ range used for approaching the surfaces during experiments and to impose an harmonic oscillation of the gap distance. The motion is vertical and the confined geometry can be immersed in a liquid bath, allowing us to work with volatile fluids.

The force sensor is a flexure hinge on which the sphere is rigidly coupled. The deflection of this elastic element and the relative displacement between the surfaces, are independently measured by two interferometers. For this purpose, one pair of mirrors is coupled to the flexure hinge and to the fixed frame of the setup and a second pair to the piezoelectric stage and to the flexure hinge. The parallelism between paired mirrors allows to reach the highest interferometric contrast. Four motors tilt 
independently each piece holding the mirrors for parallelism. For calibration or feed-back purpose, a force can be applied to the flexure hinge by a coil-magnet system located along the sphere axis.

The full setup is isolated from external vibrations. A MinusK anti-vibrating table weighted by a $350 \mathrm{~kg}$ payload filters efficiently the external mechanical vibrations above $0.5 \mathrm{~Hz}$. For thermal stability, the instrument is located in a separate room thermally insulated.

\section{B. Motion control}

The piezoelectric stage is actuated by a home made device to control the approach/retract velocity of the surfaces from $0.01 \mathrm{~nm} / \mathrm{s}$ to $10 \mathrm{~nm} / \mathrm{s}$. A voltage ramp is applied on one of the electrical terminals of the piezoelectric stack to enforce a velocity controlled displacement. We generate the voltage ramp with an integrator electrical circuit using a $10 \mu \mathrm{F}$ capacitance with very low leakage current (935C2W10K from Cornell Dubilier), a $1 \mathrm{M} \Omega$ resistance, and a high voltage operational amplifier (LTC6090) covering the voltage range $(0-120 \mathrm{~V})$ of the piezoelectric stage. The integrator system is fed by a constant voltage $V_{e}$ between $1 \mathrm{mV}$ and $1 \mathrm{~V}$, and provides a constant speed $u=\alpha V_{e} / R C$, with $\alpha=100 \mathrm{~nm} / \mathrm{V}$ the sensitivity of the piezoelectric stage.

The oscillating motion of the plane is driven by connecting the ground terminal of the piezo stage stage directly to the sinusoidal output of a SR830 lock-in amplifier. The common-mode terminal of the lock-in is connected to the common-mode of the integrator output to ensure the differential driving of the piezoelectric. The dynamic excitation of the lock-in amplifier delivers a sinusoidal voltage corresponding to a nominal oscillation amplitude from $1 \mathrm{pm}$ to $50 \mathrm{~nm}$ for the stage.

\section{Displacement sensors}

The setup contains two high-resolution displacement sensors: one measures the relative displacement of the surfaces, and the second measures the deflection of the flexure hinge in order to determine the force. Both distance measurements are based on the Nomarski interferometer, which provides a particularly high signal-to-noise ratio $^{20,21}$.

The common light source is a stabilized He-Ne laser (Melles Griot 05-STP-912 with $1 \mathrm{MHz}$ stability over 8 hours) located out of the vibration table and connected to a polarization maintaining optical fiber (Newport FPM630 FC/APC) whose output is equipped with a collimator (Newport F-H10-NIR APC) and a beam reductor. The output beam is separated into two beams of equal intensities for each interferometer, and polarized with a Glan-Taylor polarizer of high extinction ratio (Thorlabs DGL10, $100000: 1)$ at $45^{\circ}$ of the axis of a polarization separator. The latter, a modified Savart plate (MSP),

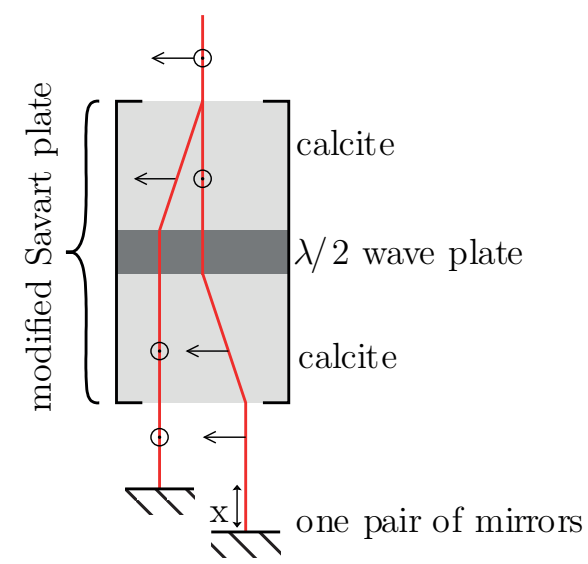

FIG. 2: Scheme of the modified Savart plate used to measure the distance $\mathrm{x}$ between two mirrors.

splits the two polarizations $\mathrm{s}$ and $\mathrm{p}$ with an equivalent optical path ${ }^{18,22}$. Each polarization pair impinges on the appropriate mirror pair and recombines in the MSP. The two output beams have an elliptical polarization corresponding to an optical phase shift $\phi=4 \pi x / \lambda+\phi_{o}$, with $x$ the distance between the two mirrors of the respective pair, and $\phi_{o}$ a residual phase. Each output beam is then sent to its own analyzing arm.

The interferometer devoted to force measurements is analyzed with the standard detection described $\mathrm{in}^{21,23}$, using a Wollaston prism and two photodiodes (Hamamatsu). The current intensities $I_{1}$ and $I_{2}$ are converted with a home-made device calculating analogically the normalized contrast:

$$
\frac{I_{1}-I_{2}}{I_{1}+I_{2}} \propto V_{o f f}+C V_{0} \cos \left(\frac{4 \pi x}{\lambda}+\phi_{0}\right)
$$

The measured contrast is higher than 0.9 , depending on the mirrors parallelism and the photodiodes gains. The amplitude $C V_{0}$ and the offset $V_{\text {off }}$ are calibrated by deflecting the flexure hinge over several wavelengths using the coil-magnet system. Due to the stiffness of the force sensor, its deflection is mainly null around the point of zero-deflection. We use a Babinet-Soleil compensator located ahead of the MSP, in order to maintain the signal around its maximum of sensitivity ${ }^{21}$ at $\phi=4 \pi x / \lambda \sim 0$.

In contrast, the interferometer measuring the relative displacement of the two surfaces has to operate over several micrometers. In order to always maintain a convenient sensitivity for any phase-shift, we use the quadrature phase set-up described by Paolino et $\mathrm{al}^{24}$. The output beam is separated into two beams, each of them analyzed as described above. In addition, one of the analyzing arm has a quarter-wave plate which adds an optical phase shift of $\pi / 2$ to the polarization. In this way two contrasts $C_{X} \simeq \cos \phi(x)$ and $C_{Y} \simeq \sin \phi(x)$ are obtained, with at least one presenting a convenient sensitivity at any $\phi(x)$ position. The quasi-static displacement is obtained by calculating numerically $\phi=$ 


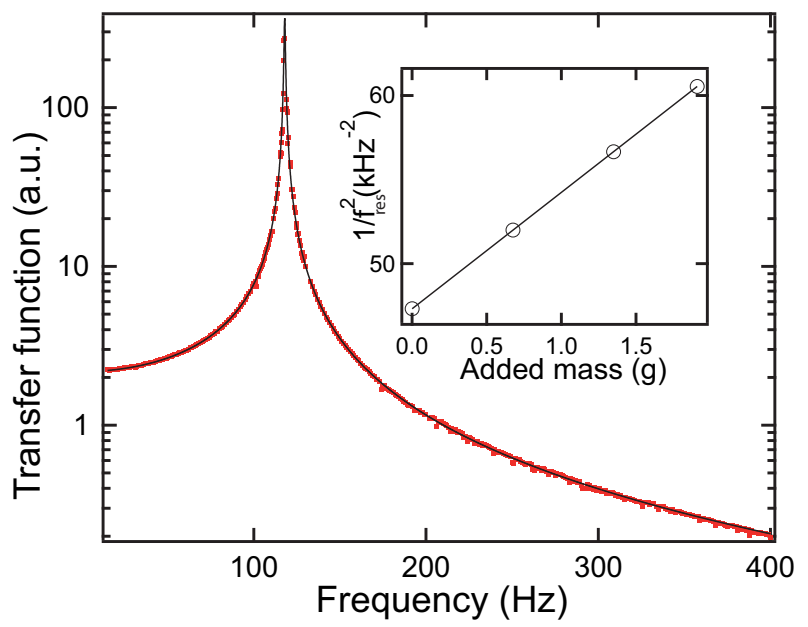

FIG. 3: Transfer function of the flexure hinge done using the coil-magnet system and two lock-in amplifiers. To obtain the transfer function, we collect the voltage corresponding to the displacement of the flexure hinge and the current going through the coil which is proportional to the force. The fit in black is based on a mass-spring model. The inset shows the so-called added mass method where some small masses are add to the flexure hinge and the resonant frequency is measured using the second order oscillator equation. The linearity between the mass and the opposite of the squared resonance frequency gives the equivalent mass $\mathrm{M}_{o}$ and the stiffness $\mathrm{K}$ of the flexure hinge

$4 \pi x / \lambda-\phi_{0}=\operatorname{atan}\left(C_{Y} / C_{X}\right)$. For the oscillatory displacement, we have developed an electronic circuit delivering in real-time a signal proportional to the small variation $\delta \phi(t)$ of the optical phase around its average value $\bar{\phi}^{25}$. The delivered signal is analyzed by a lock-in amplifier (SR830). Finally, the resolution of the sphere-plane displacement sensor, is of $0.1 \mathrm{~nm}$ for the quasi-static displacement (1 Hz bandwidth), and $1 \mathrm{pm}$ for the amplitude of the dynamic displacement.

\section{Force sensor}

The flexure hinge used as force sensor is designed using the geometry reported in ${ }^{26}$. The lateral distances between hinges are $30 \mathrm{~mm}$ and the vertical is $33 \mathrm{~mm}$. The sizes of the hinges are chosen to target a stiffness, for the vertical motion on figure 1 , of $6000 \mathrm{~N} / \mathrm{m}$. It is realized in aluminium alloy 7075 by electro-erosion. The residual motion in rotation, calculated from the actual dimensions of the hinge after realization, is of $0.5 \mu \mathrm{rad} / \mathrm{m}^{26}$. The residual rotation induces a lateral displacement of the sphere compared to the plane surface. This displacement is proportional to the sphere displacement (less than $1 \mathrm{~nm}$ for the harmonic oscillations and $100 \mathrm{~nm}$ for indentation experiments). Since the distance between the sphere and the farthest hinge, is $60 \mathrm{~mm}$, the lateral displacement is between $5.10^{-9}$ to $5 \cdot 10^{-7} \mathrm{~nm}$ depending on the sphere vertical excursion. Compared to the normal displacement, it can be neglected and the shear effects associated to the lateral displacement are not relevant in this set-up.

The mechanical response of the flexure hinge is calibrated using the coil-magnet system driven in current. The applied harmonic force $f_{d y n}$ is proportional to the current through the coils. Its dynamical response represented in figure 3 is very well described by a second order oscillator equation, with a resonance frequency $f_{\text {res }}$ $=118 \mathrm{~Hz}$ and quality factor $\mathrm{Q}=500$. The flexure hinge stiffness, measured with the method of added mass, is $\mathrm{K}=5707 \pm 0.6 \mathrm{~N} / \mathrm{m}$. The effective mass without sample is $\mathrm{M}_{o}=6.840 \pm 0.001 \mathrm{~g}$. Associated to the Nomarski interferometer, the resolution of the force sensor is $0.1 \mu \mathrm{N}$ for quasi-static forces and $10 \mathrm{nN}$ for dynamical forces.

\section{ILLUSTRATION OF THE CAPABILITIES}

We first demonstrate the possibilities of the instrument in terms of surface forces and nano-rheology measurements, on an alcane film and a visco-elastic solution confined between ultra-smooth borosilicate surfaces. The roughness of the surface is measured by taking the root mean square of the topography over an area of $100 \mu \mathrm{m}^{2}$. The rms roughness is less than $2 \AA$ on the sample presented here.

Concerning the capabilities of the instrument, we propose here few examples of possibilities. First, we will show that this rheometer is able to measure surface forces associated to van der Waals interactions, elastic indentation and adhesion in hexadecane confined between borosilicate glass. Second, we will show how to measure viscosity and the hydrodynamic boundary condition from microns to nanometers gap. Third, under very high confinement, we will show that the solid walls of the confined geometry can not be considered anymore as infinitely rigid and we will measure the response of the stack borosilicate-hexadecane in terms of elastic and viscous response. Finally we will illustrate the possibilities of the instrument to investigate the micro and nanorheological properties of a visco-elastic liquid, from the bulk to the molecular scale.

\section{A. Equilibrium surface forces}

The first example consists in confining an alcane (nhexadecane from Sigma Aldrich), between $2 \AA$ roughness floated borosilicate surfaces. The experiment is performed at room temperature $(300 \pm 1 \mathrm{~K})$, with a sphere of radius $3.24 \pm 0.02 \mathrm{~mm}$. The figure 4 represents the quasi-static force measured with respect to the sphereplane distance.

At large distance the force is bearly measurable in figure 4. At a distance smaller than $2 \mathrm{~nm}$, a negative jump of the force is observed due to the attractive Van der 
Waals forces. This attraction leads to the contact of the surfaces corresponding to a minimum of the force. This minimum is chosen as origin for the distance. The jump due to mechanical instability occurs when the negative stiffness of the attraction potential has the same absolute value as the hinge stiffness, corresponding to a force jump $\Delta F=K^{2 / 3}\left(A_{S L S} R / 3\right)^{1 / 3}$ where $A_{S L S}$ is the solid-liquid-solid Hamaker constant. From the value of the force jump $\Delta F=-4.7 \mu \mathrm{N}$, we obtain a borosilicate/hexadecane/borosilicate Hamaker constant $A_{S L S}=2.9510^{-21} \mathrm{~J}$. A full calculation of the theoretical Hamaker constant ${ }^{1}$ gives

$$
A=\frac{3}{2} k_{B} T \sum_{0}^{\infty}\left(\frac{\epsilon_{1}\left(i \nu_{n}\right)-\epsilon_{2}\left(i \nu_{n}\right)}{\epsilon_{1}\left(i \nu_{n}\right)+\epsilon_{2}\left(i \nu_{n}\right)}\right) \quad \nu_{n}=\frac{n k_{B} T}{\bar{h}}
$$

using the approximation of the optical indexes (1.433 for hexadecane and 1.5 for borosilicate) for calculating the permittivities $\epsilon_{i}\left(i \nu_{n}\right)$ of the materials at the frequency $\nu_{n}{ }^{1}$. This calculation yields a value of the Hamaker constant $A_{S L S}=2.9710^{-21} \mathrm{~J}$ in very good agreement with the measurement.

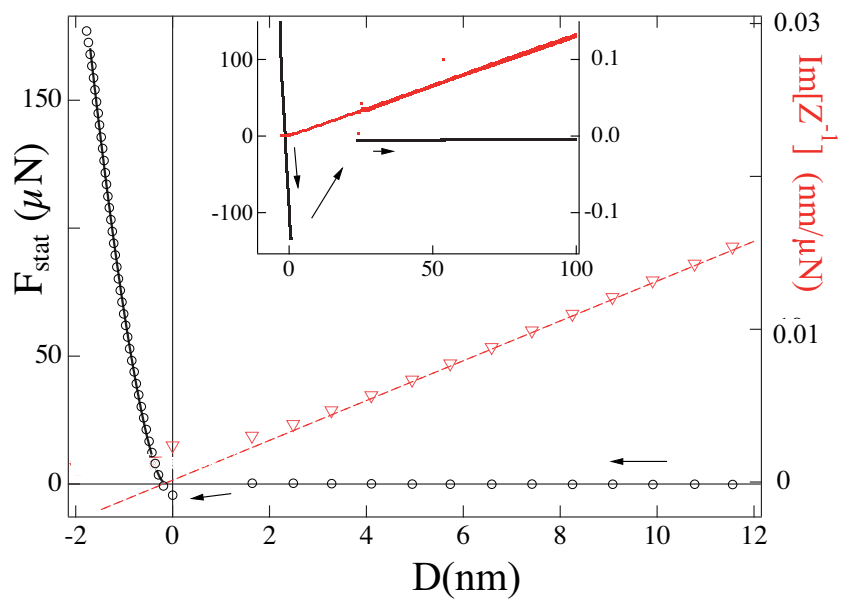

FIG. 4: Quasi-static force (o) measured on approaching borosilicate surfaces in n-hexadecane, as a function of the distance. The jump-to-contact is shown by an arrow. The origin of distance is fixed at the end of the jump. The black continuous line on $D<0$ plots the Hertz law eq. (11). Right axis $(\nabla)$ : the imaginary part of $Z^{-1}$. The dashed line is the best fit with an affine law. Inset: same quantities plotted for the backward motion.

The elastic repulsion due to indentation is measured between the surfaces for negative distance $D$ (figure 4). The force is in excellent agreement with the Hertz contact law:

$$
F_{H}=\frac{4}{3} E^{*} \sqrt{R} \delta^{3 / 2}-\Delta F
$$

where $\delta=-D, E^{*}$ is the reduced Young modulus of the two borosilicate surfaces, and $\Delta F$ accounts for the contact adhesion. The black solid line on figure 4 corresponds to the Hertz law calculated with the reduced modulus $E^{*}=34 \mathrm{GPa}$ obtained from the values of the borosilicate Young's modulus $\mathrm{E}=65 \mathrm{GPa}$ and Poisson ration $\nu=0.2$ reported by suppliers (Schott, PGO and Corning).

During the surfaces withdrawal, the force shows an important adhesion characterized by a pull-off force $F_{a d h}=135 \mu \mathrm{N}$ (inset of figure 4). Within the Derjaguin-Muller-Toporov theory appropriate for these rigid surfaces ${ }^{27}$, the pull-off force is related to the Hamaker constant and the solid-liquid surface tension by $F_{a d h} / 4 \pi R=\gamma_{S L}=A_{S L S} / 24 \pi a^{2}$ where $a$ is a molecular cut-off distance. We find a value of the hexadecaneborosilicate interfacial tension $\gamma_{S L}=3.3 \mathrm{mN} / \mathrm{m}$, and with the above value of the Hamaker constant, a molecular cut-off distance $a=1.1 \AA$. This value, slightly lower that the one reported for alcane/mica interfaces ${ }^{1}$, corroborates the ultra-low roughness of the fused borosilicate surfaces.

\section{B. Hydrodynamics and boundary condition}

The hydrodynamic impedance is measured using oscillation amplitudes $h_{o}$ between 0.01 and $40 \mathrm{~nm}$, in order to remain in a linear response domain $h_{o} / D<0.05$. The temporal signals are analyzed with two lock-in amplifiers Stanford Research SR830, one of which drives the piezoelectric stage. The lock-in amplifiers are set with a $0.3 \mathrm{~s}$ integration time and a low-pass filter decreasing with a slope of $18 \mathrm{~dB} /$ oct. The experiments are performed with a continuous drift velocity of $0.1 \mathrm{~nm} / \mathrm{s}$ close to the contact, up to $4 \mathrm{~nm} / \mathrm{s}$ at large distance. The values of the complex dynamic force and dynamic relative displacement, are numerically processed using the sensors calibration and the flexure hinge transfer function, to obtain the mechanical impedance.

The inset of figure 5 shows the inverse of the damping coefficient measured at three different frequencies of $30 \mathrm{~Hz}, 80 \mathrm{~Hz}$ and $220 \mathrm{~Hz}$ on a n-hexadecane film, as a function of the sphere-plane distance $D$. The linear variation of $1 / Z^{\prime \prime}$ as a function of the sphere-plane distance $D$ corresponds to the reference situation of eq.(2). The slopes are inversely proportional to the working frequencies, and lead to the value $\eta=2.87 \pm 0.05 \mathrm{mPa} . \mathrm{s}$ for the viscosity of hexadecane, in good agreement with the tabulated values for the bulk value of the shear viscosity of hexadecane.

The distance for which $1 / Z^{\prime \prime}$ vanishes is taken as a reference. It determines the thickness of the flowing liquid and defines the hydrodynamic origin of the distances according to eq.(2). The values of the origin found for the 3 different frequencies agree within each other, and with the mechanical origin defined from the jump-to-contact within $1 \mathrm{~nm}$. The figure 4 illustrates the location of the hydrodynamic origin. We thus conclude that hexadecane flows on borosilicate with a no-slip boundary condition 
within $1 \mathrm{~nm}$, in good agreement with the behavior found ${ }^{4}$ for the flow of alcanes on mica surfaces.

\section{Measurement of the Young modulus of borosilicate solid by confined liquid}

The elastic properties of solids can be achieved by contact measurements such as indentation, tensile testing or shear testing. The force response due to indentation depends on the elastic properties of the solid. We present here the measurement of the elastic properties of $2 \AA$ roughness borosilicate surfaces. The measurement of the Young modulus of borosilicate, without any mechanical contact, is possible due to elasto-hydrodynamic behavior of the stack borosilicate/hexadecane/borosilicate. Under high confinement, the imaginary part of $1 / \mathrm{Z}$ deviates slightly from its linear variation with the sphere-plane distance observed at large scale (figure 4, below $3 \mathrm{~nm}$ ). This deviation from the linear variation with the distance is neither a deviation from the newtonian behavior of the liquid, nor a contribution of the $2 \AA$ roughness of the surfaces. It is the signature of the deformation of the surfaces which provides an elastic response in the mechanical impedance measurements represented on the figure 5. At this scale, even borosilicate surfaces are not infinitely rigid and the deformation is measurable by liquid pressure indentation.

The figure 5 shows the components of $1 / Z$ at $220 \mathrm{~Hz}$ plotted in logarithmic scale. At a distance larger than $3 \mathrm{~nm}$, it follows the scaling laws from eq.(7), Re[1/Z] $\propto$ $D^{-1 / 2}$, which is the signature of the elasto-hydrodynamic flow of the liquid layer between semi-infinite elastic volumes. Both components are indeed in perfect agreement with the prediction without any ajustable parameter from eq.(6), using the bulk properties of hexadecane (viscosity) and borosilicate (Young's modulus and Poisson ratio) and the tabulated complex factor ${ }^{19} g_{E H}^{*}\left(D / D_{c}\right)$. At large distances, the noise observed on $\operatorname{Re}[1 / \mathrm{Z}]$ corresponds to the phase resolution of $0.2 \mathrm{mrad}$ between real and imaginary components of the lock-in amplifier.

As a consequence, this rheometer provides a very resolved measurement of the elasticity of confining media without contact, thanks to the the decay of $R e[1 / Z]$ with the distance. A Young modulus as high as $65 \mathrm{GPa}$ can be resolved using a liquid of rather low viscosity (3 mPa.s). The range of measurement of the hydrodynamic impedance extending over 5 orders of magnitude, shows the dynamical precision and the accuracy achieved by this set-up.

\section{Nano-rheology of complex liquids}

The use of the nano-rheometer to study the dynamics of a confined complex liquid is illustrated using a semi-dilute solution of poly-electrolyte in water. The poly-electrolyte is a poly-acrylamide HPAM (from SNF

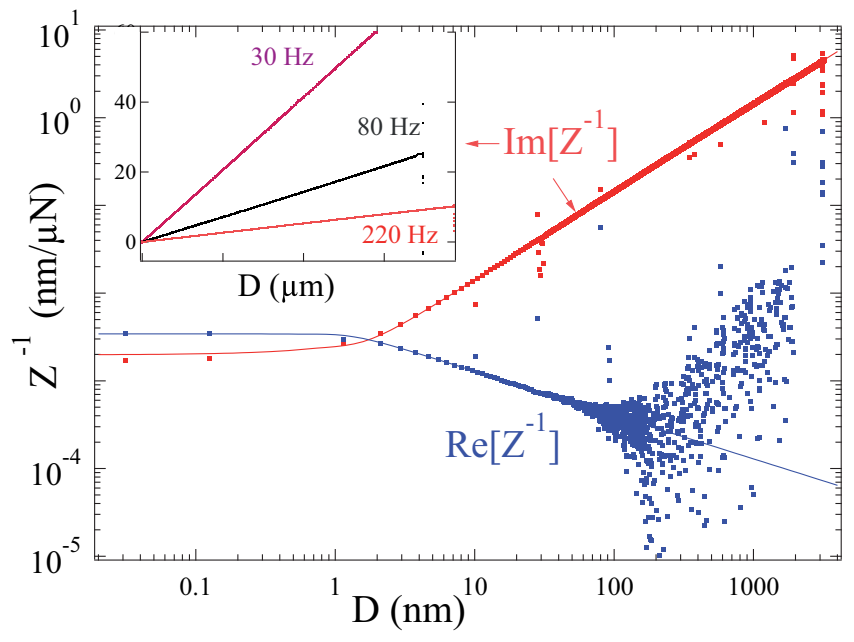

FIG. 5: Real and imaginary parts of the inverse of the hydrodynamic impedance measured at $220 \mathrm{~Hz}$ in

hexadecane between elastic borosilicate surfaces, as a function of the distance in logarithmic scale. The continuous lines are the results of the

elasto-hydrodynamic calculation extract from eq.(6) and involve no fitting parameter. The input parameters

for the calculation are the reduced Young modulus $E^{\star}=65 \mathrm{GPa}$ of borosilicate and the viscosity of hexadecane $\eta=2.87 \mathrm{mPa}$.s. Inset: Imaginary part of $Z^{-1}$ at the frequencies of $30 \mathrm{~Hz}, 80 \mathrm{~Hz}$ and $220 \mathrm{~Hz}$, plotted in linear scale as a function of the distance in micrometers.

Flopaam 3630S) of molecular weight $20.10^{6} \mathrm{~g} / \mathrm{mole}$, corresponding to chain length $1.1 \mu \mathrm{m}$. The solution is prepared with $1 \mathrm{~g}$ of dry product in 1 liter of pure water. For this concentration and molecular weigth, it is considered that the correlation length of the semi-dilute solution is about $\xi \approx 30 \mathrm{~nm}^{28}$.

The figure $6 \mathrm{a}$ plots the components of the inverse hydrodynamic impedance $Z^{-1}$ measured at $220 \mathrm{~Hz}$ over distances up to $14 \mu \mathrm{m}$. The HPAM solution has a viscoelastic behavior. At large distance $\mathrm{D}$, it viscosity $\eta$ can be extracted from the slope of $\operatorname{Im}\left[Z^{-1}\right]$. A value of $\eta$ egal to $6.6 \mathrm{mPa} . \mathrm{s}$ is measured in good agreement with bulk value for shear viscosity 28,29 . Its elastic modulus extracted from the slope of $\operatorname{Re}\left[Z^{-1}\right]$ at large $\mathrm{D}$, is $G=8.36 \mathrm{~Pa}$. It also appears on this large scale plot that the boundary condition is not a no-slip boundary condition. The dashed lines in figure $6 \mathrm{a}$ plot the best fit of the data with the Navier slip model eq. (4), using a complex slip length $b^{*}=\eta^{*} / \lambda$. The (real valued) friction coefficient $\lambda$ is the fitting parameter. We find $\lambda=31.385 \mathrm{kPa} . \mathrm{s} / \mathrm{m}$, giving a slip length $\left|b^{*}\right|=285 \mathrm{~nm}$ on each surface. The Navier slip model is in perfect agreement with the data for distances ranging from $200 \mathrm{~nm}$ to $14 \mu \mathrm{m}$. In particular, the Navier's slip model points toward the hydrodynamic origin. 

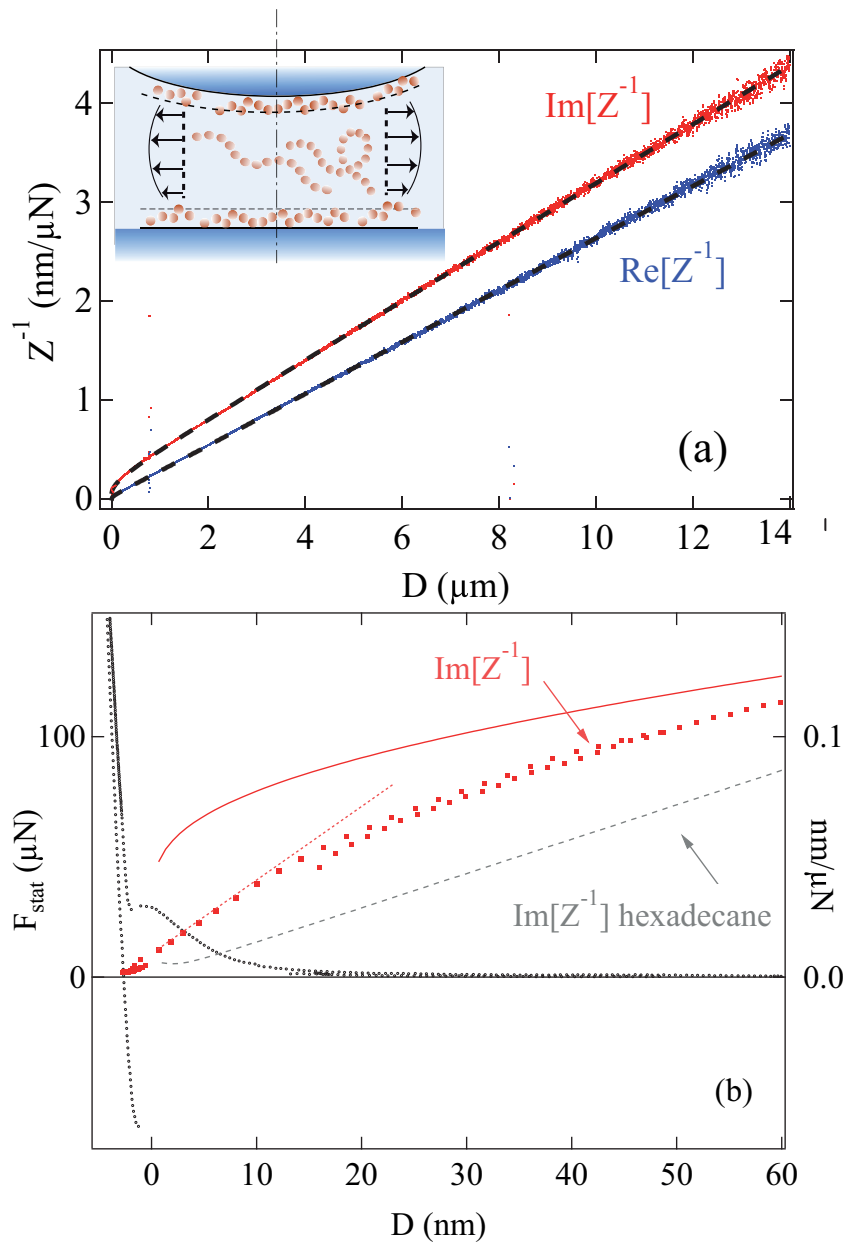

FIG. 6: (a): components of $1 / Z$ for a HPAM solution between borosilicate surfaces as a function of the distance in micrometers. The dots are the data, the dashed lines are the components of the best fit with the Navier's slip model eq. (4). (b) left axis (o): the quasi-static force between the surfaces. (b) right axis: imaginary part of $1 / Z$ (red dots). The continuous line is the best fit with the Naviers's slip model plotted in (a). The dashed gray line is the imaginary part of $1 / \mathrm{Z}$ for hexadecane.

\section{E. Revealing electrostatic depletion}

At a lower scale (figure 6b) the Navier's slip model deviates from the data and predicts a lower hydrodynamic force than the measured one. Below $20 \mathrm{~nm}$, the quasistatic force increases exponentially, showing an electrostatic repulsion between the surfaces. The associated surface charge, of about $20 \mathrm{mC} / \mathrm{m}^{2}$, is much larger than the charge of borosilicate surfaces in water and is attributed to an adsorption layer on the surfaces. The polymer chains in the solution, of same charge than the adsorption layer, are then repelled out of the Electrostatic Double Layer (EDL), resulting in a depletion layer of about 15 $\mathrm{nm}$ on each surface, as shown on the profile of the static force. The depletion layer has a viscosity lower than the bulk, as shown by the slope of $\operatorname{Im}\left[\mathrm{Z}^{-1}\right]$ as a function of $D$ in the EDL region sketched in figure $6 a$. Thus we can interpretate the slippage observed at large distance as the effect of an electrostatic depletion layer. This original result opens a new framework for the interpretation of slippage of fluid onto surfaces: a 2-fluid model incorporating a depletion layer of finite thickness on each wall would be more appropriate than the Navier slip model to account for the rheological behavior of the solution at confinements below $200 \mathrm{~nm}$.

\section{v. CONCLUSION}

A micro-nano-rheometer dedicated to mechanical studies of soft interfaces and confined media has been described. Two interferometric sensors of high resolution give simultaneous access to both the separation and the force between the surfaces with a range of distance from 1 $\mathrm{nm}$ to 10 micrometers, and a frequency range from $10 \mathrm{~Hz}$ to $400 \mathrm{~Hz}$. The use of the device to bridge macroscopic to molecular rheological properties of liquids at interfaces is demonstrated on two examples involving visco-elasticity, boundary slippage, and elasto-hydrodynamic measurements.

\section{ACKNOWLEDGMENTS}

We are indebted to Frédéric Restagno for helpful discussions. We thank Jean-Luc Mocellin and Bruno Travers for the technical assistance on electronic conception. This work was partially supported by the ARN Grant ANR-15-CE06-0005-02 and by CNRS Defi Instrumentation. Additionally, we are grateful to the COST action MP1303.

\footnotetext{
${ }^{1} \mathrm{~J}$. Israelachvili, Intermolecular and Surface Forces, 2nd ed. (Academic Press, London, 1992).

${ }^{2}$ J. Israelachvili and G. Adams, Nature 262, 774 (1976).

${ }^{3}$ R. Horn and J. Israelachvili, J. Chem. Phys. 75, 1400 (1981).

${ }^{4}$ D. Y. C. Chan and R. G. Horn, J. Chem. Phys. 83, 5311 (1985).

${ }^{5}$ P. Richetti and P. Kekicheff, Phys. Rev. Lett. 68, 1951 (1992).

${ }^{6}$ A. Dhinojwala and S. Granick, Macromolecules, Macromolecules 30, 1079 (1997).

${ }^{7}$ U. Raviv, P. Laurat, and J. Klein, Nature 413, 51 (2001).

${ }^{8}$ Y. Zhu and S. Granick, Phys. Rev. Lett. 88, 106102 (2002).

${ }^{9}$ B. Cross, A. Steinberger, C. Cottin-Bizonne, J. P. Rieu, and E. Charlaix, Europhys. Lett. 73, 390 (2006).

${ }^{10}$ A. Steinberger, C. Cottin-Bizonne, P. Kleimann, and E. Charlaix, Phys. Rev. Lett. 100, 134501 (2008).

${ }^{11}$ S. Leroy, A. Steinberger, C. Cottin-Bizonne, F. Restagno, L. Léger, and E. Charlaix, Phys. Rev. Lett. 108, 264501 (2012).

${ }^{12}$ S. Carpentier, M. S. Rodrigues, E. Charlaix, and J. Chevrier, Applied Physics Lett. 107, 044101 (2015).

${ }^{13}$ H. Brenner, Chem. Eng. Sci. 16, 242 (1961).

${ }^{14}$ E. Pelletier, J.-P. Montfort, and F. Lapique, Journal of Rheology 38, 1151 (1994).

${ }^{15}$ C. Navier, Mem. Acad. Sci. Inst. Fr. 6, 389 (1823).

${ }^{16}$ L. Hocking, J. of Engineering Mathematics 7, 207 (1973).

${ }^{17}$ O. Vinogradova, Langmuir 11, 2213 (1995).
} 
${ }^{18}$ C. Barraud, Ph.D. thesis, Université Grenoble Alpes, Grenoble, France (2016).

${ }^{19}$ S. Leroy and E. Charlaix, J. Fluid Mech. 674, 389 (2011).

${ }^{20}$ G.Nomarski, J. Phys. Radium 16, 95 (1955).

${ }^{21}$ C. Schonenberger and S. F. Alvarado, Review of Scientific Instruments 60, 3131 (1989).

${ }^{22}$ N. Bello Gonzalez and F. Kneer, Astronomy and Astrophysics 480, 265 (2008).

${ }^{23}$ F. Restagno, J. Crassous, É. Charlaix, C. Cottin-Bizonne, and M. Monchanin, Review of Scientific Instruments 73, 2292 (2002).
${ }^{24}$ P. Paolino, F. A. A. Sandoval, and L. Bellon, Rev. Sci. Instrum. 84, 095001 (2013).

${ }^{25}$ C. Barraud, L. Garcia, B. Cross, and E. Charlaix, submitted to Measurement Science and Technology (2016).

${ }^{26}$ S. T. Smith, D. G. Chetwynd, and D. K. Bowen, Journal of Physics E: Scientific Instruments 20, 977 (1987).

${ }^{27}$ B. V. Derhaguin, V. M. Muller, and Y. P. Toporov, J. Coll. Interf. Sci. 53, 314 (1975).

${ }^{28}$ A. Cuenca and H. Bodiguel, Phys. Rev. Lett. 110, 108304 (2013).

${ }^{29} \mathrm{C}$. Gao, Journal of Petroleum Exploration and Production Technology 3, 203 (2013). 